\title{
Diffuse traumatic brain injury affects chronic corticosterone function in the rat
}

\author{
Rachel K Rowe ${ }^{1,2,3}$, Benjamin M Rumney, ${ }^{2,3,4}$, Hazel G May, 2,3, , Paska Permana1, \\ P David Adelson 2,3,5, S Mitchell Harman', Jonathan Lifshitz ${ }^{1,2,3}$ and \\ Theresa C Thomas ${ }^{1,2,3}$ \\ ${ }^{1}$ Phoenix Veterans Affairs Health Care System, Phoenix, Arizona, USA \\ 2BARROW Neurological Institute at Phoenix Children's Hospital, Phoenix, Arizona, USA \\ ${ }^{3}$ Department of Child Health, University of Arizona College of Medicine - Phoenix, Phoenix, Arizona, USA \\ ${ }^{4}$ Department of Biology and Biochemistry, University of Bath, UK \\ ${ }^{5}$ School of Biological and Health Systems Engineering, Arizona State University, Tempe, Arizona, USA
}

\author{
Correspondence \\ should be addressed \\ to T C Thomas \\ Email \\ theresathomas@email. \\ arizona.edu
}

\begin{abstract}
As many as $20-55 \%$ of patients with a history of traumatic brain injury (TBI) experience chronic endocrine dysfunction, leading to impaired quality of life, impaired rehabilitation efforts and lowered life expectancy. Endocrine dysfunction after TBI is thought to result from acceleration-deceleration forces to the brain within the skull, creating enduring hypothalamic and pituitary neuropathology, and subsequent hypothalamic-pituitary endocrine (HPE) dysfunction. These experiments were designed to test the hypothesis that a single diffuse TBI results in chronic dysfunction of corticosterone (CORT), a glucocorticoid released in response to stress and testosterone. We used a rodent model of diffuse TBI induced by midline fluid percussion injury (mFPI). At 2 months postinjury compared with uninjured control animals, circulating levels of CORT were evaluated at rest, under restraint stress and in response to dexamethasone, a synthetic glucocorticoid commonly used to test HPE axis regulation. Testosterone was evaluated at rest. Further, we assessed changes in injury-induced neuron morphology (Golgi stain), neuropathology (silver stain) and activated astrocytes (GFAP) in the paraventricular nucleus (PVN) of the hypothalamus. Resting plasma CORT levels were decreased at 2 months postinjury and there was a blunted CORT increase in response to restraint induced stress. No changes in testosterone were measured. These changes in CORT were observed concomitantly with altered complexity of neuron processes in the PVN over time, devoid of neuropathology or astrocytosis. Results provide evidence that a single moderate diffuse TBI leads to changes in CORT function, which can contribute to the persistence of symptoms related to endocrine dysfunction. Future experiments aim to evaluate additional HP-related hormones and endocrine circuit pathology following diffuse TBI.
\end{abstract}

\author{
Key Words \\ - TBI \\ - concussion \\ - corticosterone \\ stress
}

Endocrine Connections (2016) 5, 152-166

\section{Introduction}

Traumatic brain injury (TBI) is a major cause of death and disability throughout the world with few pharmacological treatments for the individuals who suffer from lifelong neurological symptoms associated with TBI. Brain injuries are the predominant injury of soldiers participating in Operation Iraqi Freedom

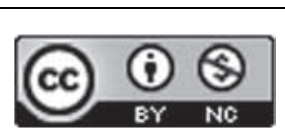

This work is licensed under a Creative Commons Attribution-NonCommercial 4.0 International License. 
(OIF) and Operation Enduring Freedom (OEF) combat operations, with $46 \%$ screening positive for TBI (1). TBI is initiated by the unexpected impact of mechanical force(s) to the head or brain. The ensuing postinjury cellular cascades lead to the development of pathological processes over time (2). Onset of symptoms begins within minutes to hours after impact and can contribute to the development of long-lasting neurological impairments. Clinically prevalent symptomology includes somatic, cognitive and emotional impairments, with chronic endocrine dysfunction becoming increasingly recognized as a clinical issue $(3,4,5)$.

Endocrine dysfunction after TBI can be attributed to the acceleration and deceleration of the brain inside the skull resulting in neuropathology, including hemorrhage, necrosis and fibrosis of the pituitary, infundibulum and hypothalamus (6). Functionally, the pituitary is the 'master gland' controlling growth, reproduction, adrenal regulation, water balance and thyroid function through secretion of trophic peptides into the circulatory system. Together, the hypothalamus and pituitary form the central component of the hypothalamicpituitary-endocrine (HPE) axis. Structural damage to these regions can contribute to the manifestation of transient and chronic endocrine dysfunction along with other associated clinical phenotypes, indicative of TBI being a chronic disease process rather than an acute event $(2,7,8)$.

Chronic endocrine dysfunction can be defined as long-lasting changes in the production, release, circulation and/or regulation of hormones that result in one or more symptoms including emotional liability, anxiety, aggressiveness, sexual dysfunction, abdominal weight gain, memory impairment, lethargy and decreased stamina (9). In as many as $20-55 \%$ of patients with a history of TBI $(10,11,12,13,14,15)$, chronic endocrine dysfunction is sufficiently severe enough to impair quality of life and impede rehabilitation efforts $(6,16)$, and in rare instances, increases risk for mortality $(17,18$, 19). Pituitary hormone deficiencies or hypopituitarism, to a limited extent, are reported in TBI survivors, but the presentation of clinical symptoms depend heavily on the magnitude of specific hormone deficiency $(20,21,22)$. As such, TBI survivors can present with deficiencies in one or more hormones, including corticotropins, growth hormones, thyrotropins, and gonadotropins (20). The variation of hormones disrupted could be based on the type (blast, diffuse, penetrating), mechanics (direction/force of impact) or dynamics (single, repetitive) of the injury, as well as pre-existing conditions (genetic predisposition) $(23,24,25,26,27,28)$. Recent longitudinal studies support the idea that a severe injury is more likely to result in chronic endocrine dysfunction, as opposed to a mild injury; however, endocrine dysfunction manifests across the brain injury severity spectrum (15). Injuryinduced endocrine dysfunction can be concomitant with and potentially augment clinical symptoms commonly associated with postconcussive syndrome and posttraumatic stress disorder (PTSD) $(22,29)$. While the prevalence of endocrine dysfunction is reported in the clinical literature, few experimental models have elucidated the persistence and pathological basis of mild-moderate diffuse brain injury-induced endocrine dysfunction $(9,30)$.

Previous data from our laboratory indicate that under normal conditions, a non-noxious behavioral task does not increase levels of corticosterone (CORT), a steroid released by the adrenal glands in response to adrenocorticotropin hormone (ACTH) from the anterior pituitary (31). However, rats with mild to moderate diffuse TBI respond to the non-noxious whisker nuisance task (WNT) with a change in the allodynic response accompanied by increases in levels or corticosterone (CORT). Other investigators also report CORT dysregulation following focal brain injury using controlled cortical impact (CCI) $(32,33,34,35)$. These data support that rodents can experience endocrine dysfunction after TBI. The following experiments were designed to develop this profile of endocrine dysfunction in a rodent model of diffuse brain injury induced by midline fluid percussion. Further, we assessed changes in injury-induced neuron morphology, neuropathology (silver stain), and astrocyte activation (GPAP) in the paraventricular nucleus (PVN) of the hypothalamus, a region responsible for initiating the release of CORT from the adrenal glands via corticotropin-releasing hormone $(\mathrm{CRH})$ and ACTH from the anterior pituitary. Multiple assessments (i.e. hormonal, behavioral and histological) were made at chronic time points postinjury to determine whether ongoing reparative processes could contribute to late-onset deficits. We hypothesized that diffuse TBI would lead to chronic deficiencies in CORT and testosterone, which are regulated by the hypothalamic-pituitaryadrenal axis and hypothalamic-pituitary-gonadal axis, respectively. Furthermore, we predicted an increased sensory sensitivity in brain-injured rats assessed by the whisker nuisance task (31). Finally, we predicted ongoing pathology in PVN neurons of the hypothalamus. 


\section{Methods}

\section{Study design}

The following experiments used four cohorts of rats. Cohort one was used to assess midline fluid percussion injury (mFPI) on weight change, sensory sensitivity measured by the whisker nuisance task, neuroendocrine hormone levels of CORT and testosterone and dexamethasone treatment. Cohorts two, three and four were used to assess brain injury-induced changes to the PVN following mFPI using Golgi stain, silver stain and GFAP immunohistochemistry. All animal studies were conducted in accordance with the guidelines established by the internal IACUC (Institutional Animal Care and Use Committee) and the NIH guidelines for the care and use of laboratory animals. Studies are reported following the ARRIVE (Animal Research: Reporting In Vivo Experiments) guidelines. Randomization of animals was achieved by assigning animals to treatment groups before the initiation of the study to ensure equal distribution across groups. A power analysis was performed to calculate group sizes to enable statistically robust detection of injury-induced deficits while minimizing the number of animals. This calculation was based on preliminary data and previously published work from our group. Data collection stopped at predetermined final endpoints based on days postinjury (DPI) for each animal. Four animals were excluded from the study because postoperative weight decreased by $10 \%$ of presurgical weight or visible signs of pain or discomfort beyond 5 DPI were observed. All animal behavior was scored by investigators blinded to the treatment groups. Data sets were screened using the extreme studentized deviate method for significant outliers.

\section{Animals}

Adult male Sprague Dawley rats (Harlan Laboratories, Inc., Indianapolis, IN) were used for all experiments. Rats were housed in a $12 \mathrm{~h}$ light: $12 \mathrm{~h}$ darkness cycle $(06: 00 / 18: 00 \mathrm{~h})$ at a constant temperature $\left(23 \pm 2^{\circ} \mathrm{C}\right)$ with food and water available ad libitum according to the Association for Assessment and Accreditation of Laboratory Animal Care International. Rats were acclimated to their environment following shipment for at least 1 week before any experiments. After surgery, rats were evaluated daily for postoperative care by a physical examination and documentation of each animal's condition. (c) 2016 The authors Published by Bioscientifica Ltd.

\section{Midline fluid percussion injury (mFPI)}

Rats (350-370 g) were subjected to midline fluid percussion injury (mFPI) consistent with methods described previously $(31,36,37,38,39)$. Rats were anesthetized using 5\% isoflurane in 100\% oxygen for $5 \mathrm{~min}$ and the head of the rat was placed in a stereotaxic frame with continuously delivered isoflurane at $2.5 \%$ via nosecone. While anesthetized, body temperature was maintained using a Deltaphase isothermal heating pad (Braintree Scientific Inc, Braintree, MA, USA). A midline incision was made exposing bregma and lambda, and fascia was removed from the surface of the skull. A trephine $(4.8 \mathrm{~mm}$ outer diameter) was used for the craniotomy, centered on the sagittal suture between bregma and lambda without disruption of the dura. An injury cap prepared from the female portion of a Luer-Loc needle hub was fixed over the craniotomy using cyanoacrylate gel and methylmethacrylate (Hygenic Corp, Akron, OH, USA). The incision was sutured at the anterior and posterior edges and topical Lidocaine ointment was applied. Rats were placed in a heated recovery cage and monitored until ambulatory.

For injury induction, rats were reanesthetized (60-90 min after surgery) with 5\% isoflurane delivered for $5 \mathrm{~min}$. The dura was visually inspected through the hub to make sure it was intact with no debris. The hub was then filled with normal saline and attached to the male end of the fluid percussion device (Custom Design and Fabrication, Virginia Commonwealth University, Richmond, VA, USA). An injury of moderate severity for our injury model $(2.1 \mathrm{~atm})$ was administered by releasing the pendulum onto the fluid-filled cylinder. Sham-injured rats underwent the same surgical procedures except the pendulum was not released. Rats were monitored for the presence of a forearm fencing response, and righting reflex times were recorded for the injured rats as indicators of injury severity (40). The righting reflex time is the total time from the initial impact until the rat spontaneously rights itself from a supine position. The fencing response is a tonic posturing characterized by extension and flexion of opposite arms that has been validated as an overt indicator of injury severity (40). The injury hub was removed and the brain was inspected for uniform herniation and integrity of the dura. The dura was intact in all rats; none were excluded as technical failures. The incision was cleaned using saline and closed using sutures. Moderate brain-injured rats had righting reflex recovery times greater than $6 \mathrm{~min}$ and a positive fencing response. Sham-injured rats recovered a righting

This work is licensed under a Creative Commons Attribution-NonCommercial 4.0 International License.

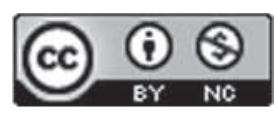

http://www.endocrineconnections.org DOI: 10.1530/EC-16-0031 
reflex within 20s. After spontaneously righting, rats were placed in a heated recovery cage and monitored until ambulatory (approximately 5-15 min) before being returned to their cage. Adequate measures were taken to minimize pain or discomfort.

\section{Post-operative weights}

The weights of each rat were recorded on the day of surgery. Following surgery, animals were postoperatively monitored for the first 3 days postinjury. This monitoring included a physical examination, thorough examination of the suture site and evaluation of changes in postoperative weight. Before tissue collection, terminal weights were recorded at $58 \mathrm{DPI}$.

\section{Behavioral testing}

Whisker nuisance task Protocols were conducted as described previously $(31,41)$, with the exception of baseline whisker nuisance scores being collected before surgical procedures to induce mFPI. A plastic test cage $(16.5 \times 38.1 \times 55.9 \mathrm{~cm})$ lined with an absorbent pad was used. Rats were acclimated to the test cage for $5 \mathrm{~min}$ before testing. Testing involved manually stimulating the whiskers of both mystaical pads with a wooden applicator stick for three periods of $5 \mathrm{~min}$ with $\leq 1 \mathrm{~min}$ of nonstimulation between periods. Animals were tested individually and the absorbent pad and wooden applicator stick were replaced for each animal after cleaning the test cage. For each 5-min period, observations were made regarding the predominant observed behavior. These observations were categorized as (i) movement, (ii) stance and body position, (iii) breathing quality, (iv) whisker position, (v) whisking response, (vi) evading stimulation, (vii) response to stick presentation and (viii) grooming response. Normal behavior for each category consisted of those typically seen in uninjured animals during stimulation and was recorded as a score of zero. Meaningful abnormal behaviors expressed in response to whisker stimulation were assigned scores of 1-2, depending on degree of expression. The maximum whisker nuisance score was 16 (two points for each of eight categories). Higher scores indicate abnormal responses to the stimulation and lower scores indicate normal responses.

\section{Restraint stress and blood collection}

A preinjury blood sample (baseline) was taken from each rat before mFPI or sham injury. The day of surgery (between 08:45 and 12:00 h), rats were removed from their cage and anesthetized for $5 \mathrm{~min}$ with isoflurane. Immediately following anesthesia, rats were secured in a stereotaxic frame and blood collection $(250 \mu \mathrm{L})$ through a tail vein occurred. At 54 and 56 DPI, rats underwent restraint stress and blood sampling. Samples were obtained in the acute period immediately following light onset (initiated between 07:30 and 09:50 h) to control for corticosterone having diurnal peaks. A rat was transported in its home cage to procedure room, and placed tightly into flat-bottom Plexiglas restraining tubes (Braintree Scientific Inc, Braintree, MA, USA). The process took $\sim 2-6$ min from when the animal was disturbed until the first blood draw was acquired. If the initial blood draw required multiple attempts, the animal was removed from the study. Rats remained in the restrainer for a total of $90 \mathrm{~min}$ (30 min of tight restraint, followed by $60 \mathrm{~min}$ of loosened restraint) and blood was repeatedly collected $(250 \mu \mathrm{L})$ through tail vein venipuncture at 30, 60 and $90 \mathrm{~min}$, similar to previously published methods (33, 35). The restrainer (head access rodent restrainer, Stoelting, Wood Dale, IL, USA) can be adjusted to four chamber sizes inducing varying levels of restraint. In the current study, tight restraint was defined by the smallest chamber size in which the animal could not adjust themselves. Loose restraint was defined by the second largest chamber size in which the animal could not turn around but could freely adjust body position. All blood collection occurred between 08:00 and 12:00 h. Blood samples were collected into tubes coated with EDTA and kept on ice until centrifuged at $1300 \boldsymbol{g}$ for $10 \mathrm{~min}$. Each plasma sample was divided into three labeled tubes and stored at $-20^{\circ} \mathrm{C}$ until processed further.

\section{Dexamethasone treatment}

At 56 DPI, rats were administered a subcutaneous dose of $0.1 \mathrm{mg} / \mathrm{kg}$ dexamethasone (DEX) (Dexamethasone Injectable sc-362917Rx, Santa Cruz Biotechnology) at approximately $06: 00 \mathrm{~h}$ to investigate effectiveness of HPA axis negative feedback mechanisms (42). This dose was guided by a literature review of rats receiving DEX before or following experimental brain injury (33, 43, $44,45)$. Two hours postinjection, the rats were placed in restrainers and the restraint stress paradigm was repeated under the influence of DEX (see the 'Restraint stress and blood collection' section above). 


\section{Testosterone and corticosterone}

Plasma testosterone and CORT levels were quantified using ELISA kits purchased through Enzo Life Sciences, Inc (Farmingdale, NY, USA). CORT samples were run in triplicate and testosterone samples in duplicate following the manufacturer's instructions. Plasma samples were diluted 1:50 per manufacturer's recommendations for the CORT ELISA. Samples with the DEX treatment were run at higher concentrations (experiments were repeated at dilutions of 1:10-1:25). Samples for the testosterone ELISA were diluted 1:20, per manufacturer's instructions.

\section{Morphological analysis on Golgi-stained tissue}

At $1(n=4), 7(n=4)$ and $28(n=3)$ DPI, sham- $(n=4)$ and brain-injured rats were overdosed with sodium pentobarbital (200 mg/kg, i.p.), decapitated and the brain was rapidly removed, rinsed with $\mathrm{ddH}_{2} \mathrm{O}$, grossly dissected into three pieces (anterior to bregma, posterior to bregma and cerebellum). At least one sham-operated animal was used at each time point, and combined into one sham cohort. Tissue was processed for Golgi staining using manufacturer's instructions provided in the FD Rapid GolgiStain Kit (FD NeuroTechnologies, Inc, Columbia, MD, USA). Brains were mounted on chucks with frozen $\mathrm{ddH}_{2} \mathrm{O}$ and then sectioned $(200 \mu \mathrm{m})$ at $-22^{\circ} \mathrm{C}$ on a cryostat and mounted on 3\% gelatin-coated microscope slides. The slides were then stained according to FD Rapid GolgiStain protocol. The slides were cleared in xylene and cover-slipped with Permount.

Changes in PVN neuronal dendritic morphology were assessed over DPI using Neurolucida software (MicroBrightField, Inc, Colchester, VT, USA). Neurons were traced in three dimensions (3D; $\mathrm{x}, \mathrm{y}, \mathrm{z})$ by an investigator blinded to injury status. All neurons chosen for reconstruction had an intact soma and the presence of natural ends (termination of a completely tapered dendrite, which can be identified by a spine or spine cluster or gradual narrowing). Neurons were chosen based on neuron cell morphology and discriminate staining margins of the neuron cell body and processes. All neuronal processes and soma were traced at $40 \times$ magnification, tracing the boundaries of each cell soma and then all emerging processes to their full extent. 3D reconstructions were analyzed using NeuroExplorer software (MicroBrightField, Inc, Colchester, VT, USA) to determine process quantity, branch points, process complexity index ((sum of terminal orders + number of terminals $) \times$ (total process length/number of primary processes)) (adapted from Pillai et al. 2012 (46)) and overall process complexity (Sholl analysis). Parvocellular neurons, which were traced, were differentiated from magnocellular neurons based on localization within the medial PVN, which was demarcated by the 3rd ventricle and fornix $(47,48,49,50,51)$. Ten neurons from this area were traced per animal and averaged for a single representative number per animal, resulting in a total of 150 neurons being traced. Sholl analysis was performed to evaluate the distribution of dendritic processes. A series of concentric spheres with increasing radius $(10 \mu \mathrm{m})$ centered on the soma was used to assess the number of process crossings as a function of distance and space using NeuroExplorer software. Image z-stacks were carried out in $1 \mu \mathrm{m}$ increments from the top-most process to the bottom-most process and deep-focused. The 3D reconstructions from Neurolucida software were inverted and converted to black and white in Photoshop (Adobe CS6).

\section{Quantification of silver stain in the paraventricular nucleus}

The de Olmos aminocupric silver histochemical technique $(52,53,54)$ was carried out to assess neuropathology after diffuse TBI. At designated times, sham- $(n=3$; one per time point) or brain-injured rats ( $n=3$ per time point) were overdosed with sodium pentobarbital (200 mg/kg i.p.) and transcardially perfused with $0.9 \%$ sodium chloride, followed by a fixative solution containing $4 \%$ paraformaldehyde and $4 \%$ sucrose in $0.1 \mathrm{M}$ phosphatebuffered saline. Fixative solution containing 15\% sucrose was used to store the heads for $24 \mathrm{~h}$ after decapitation. The brains were extracted, placed in fresh fixative and shipped to Neuroscience Associates Inc (Knoxville, TN, USA). Brains were embedded into a single gelatin block (Multiblock Technology; Neuroscience Associates, Knoxville, TN, USA), cryosectioned, mounted and stained with the de Olmos aminocupric silver staining technique in accordance to proprietary protocols (Neuroscience Associates, Knoxville, TN, USA). The argyrophilic reaction product displayed neurons and neuronal processes, which were counterstained with Neutral Red and subsequently cover-slipped. The stained sections were analyzed in our laboratory similar to previously published anatomical regions (55).

All silver-stained slides were photographed using a Zeiss microscope (Imager A2) in bright-field mode 
with a digital camera (AxioCam MRc5). A densitometric quantitative analysis was performed on all argyrophilic tissue staining at $20 \times$ magnification using ImageJ software (1.48v, NIH, Bethesda, MD, USA). Grayscale digital images were digitally thresholded to separate positive-stained pixels from unstained pixels, and then segmented into black and white pixels, indicative of positive and negative staining, respectively. The percentage of argyrophilic (black) staining was calculated using the following formula:

$\frac{\text { Total Area Measured Black }}{\text { Total Area Measured }} \times 100=$ Percentage Area with Argyrophilic Stain

Four images were analyzed per animal, averaged to a single number and used for statistical analysis.

\section{GFAP}

At $1(n=3), 7(n=4)$ and $28(n=3)$ DPI, sham- $(n=4)$ and brain-injured rats were overdosed with sodium pentobarbital and transcardially perfused with PBS and $4 \%$ paraformaldehyde. Brains were immersed in serial sucrose dilutions $(15 \%, 30 \%)$ and cryosectioned $(20 \mu \mathrm{m})$. Sections were washed $3 \mathrm{x}$ with 1 XPBS, blocked with $4 \%$ $\mathrm{v} / \mathrm{v}$ normal horse serum in PBS for $1 \mathrm{~h}$, incubated in the primary antibody (rabbit anti-GFAP, 1:1000; Dako, catalog\# Z0334) overnight at room temperature, rinsed in 1XPBS, incubated for $1 \mathrm{~h}$ at room temperature with the secondary antibody (biotinylated horse antirabbit, 1:250; Vector Laboratories, Burlingame, CA, USA; Catalog\# BA-1100), washed, blocked with $0.3 \%$ hydrogen peroxide, incubated with avidin/biotin complex $\left(4^{\circ} \mathrm{C}, 20 \mathrm{~min}\right)$, washed and incubated with DAB (10 min). The sections were dehydrated with ethanol, cleared in citrisolv and cover-slipped using DPX. Densitometric quantitative analysis was performed identical to silver stain. Four to six images were analyzed per animal, averaged to a single number and used for statistical analysis.

\section{Statistical analysis}

Data are shown as mean \pm s.e.M. and analyzed using statistical software (GraphPad-Prism 6). Differences in testosterone and CORT baseline and 54 DPI levels were measured one-way ANOVA followed by a Tukey's multiple comparison test. Changes in CORT following stress and DEX in combination with stress were analyzed using a repeated measure two-way ANOVA. Nonparametric whisker nuisance task data were analyzed using a Mann-Whitney test. The average neuron process quantity, branch points and process complexity index were subjected to statistical analysis by one-way ANOVA with a Tukey's post hoc comparison. Golgi-stained morphology data were analyzed as a function of days postinjury and distance from soma using a two-way ANOVA with a Tukey's post hoc comparison (Sholl analysis). Averages of the percent area of argyrophilic staining and GFAP within the paraventricular nucleus were compared using a one-way ANOVA. Differences in weight change were compared by Student's $t$-test. Statistical significance was assigned when $P<0.05$.

\section{Results}

\section{Diffuse TBI resulted in decreased CORT but not testosterone at 54 DPI}

Tail vein blood was collected at the time of surgery (05:00-09:00 h) from all rats (baseline levels). Diffuse TBI significantly decreased plasma CORT levels measured at 54 DPI compared with uninjured shams and baseline levels $(\mathrm{F}(2,23)=5.251, P=0.0132$; Fig. $1 \mathrm{~A})$. There was no significant difference in plasma testosterone levels in the brain-injured group compared with uninjured shams and baseline levels $(\mathrm{F}(2,29)=1.306, P=0.2942$; Fig. 1B $)$.

\section{Diffuse TBI resulted in a blunted CORT response under stressed conditions}

CORT levels were measured at 0 min (immediately after being placed in a restrainer), 30, 60 and $90 \mathrm{~min}$ postinduction of restraint. Both TBI and sham groups demonstrated increased CORT levels in response to restraint stress over time, indicated by a significant time
A

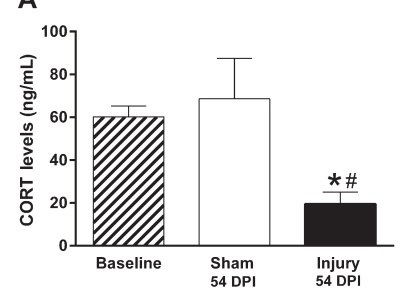

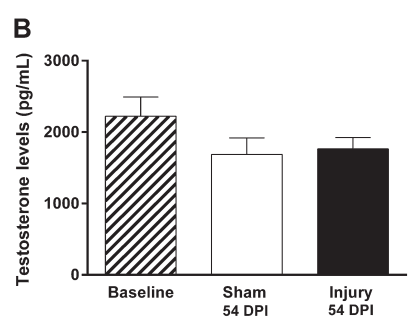

Figure 1

Diffuse TBI resulted in decreased CORT but not testosterone at $54 \mathrm{DPI}$. At the time of surgery, baseline blood was collected from all rats. At 54 DPI, before stress, blood was collected. (A) Diffuse brain injury decreased plasma CORT levels in brain-injured rats compared with uninjured shams and baseline levels at 54 DPI. (Data presented as mean \pm s.E.M.; sham $n=6$, injured $\left.n=6,{ }^{*} P<0.05\right)$. (B) There were no significant differences in plasma testosterone levels $54 \mathrm{DPI}$ compared with uninjured shams and baseline levels. (Data presented as mean \pm S.E.M.; sham $n=6$, injured $n=5$ ).

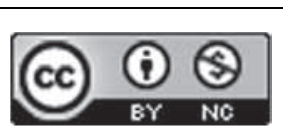

This work is licensed under a Creative Commons Attribution-NonCommercial 4.0 International License. 
effect $(F(3,33)=26.80, P<0.0001$; Fig. 2A). In addition, there was an overall significant injury effect on CORT levels compared with uninjured shams $(F(1,11)=4.946$, $P=0.0480$; Fig. 2A). The brain-injured rats had a blunted CORT response to restraint stress, and post hoc analysis indicated brain-injured rats had significantly lower CORT levels at $60 \mathrm{~min}$ poststress induction, compared with uninjured shams.

\section{DEX suppressed CORT levels in brain-injured rats and uninjured shams under stressed conditions}

Two hours before restraint stress at 56 DPI, rats were subcutaneously injected with DEX $(0.1 \mathrm{mg} / \mathrm{kg})$. Following DEX treatment, rats underwent the restraint stress paradigm (see methods). Following DEX treatment and restraint stress, there were no injury-induced differences in plasma CORT levels compared with uninjured shams $(\mathrm{F}(1,10)=4.337, P=0.0639$; Fig. 2B). The DEX suppressed CORT even under stressed conditions indicated by
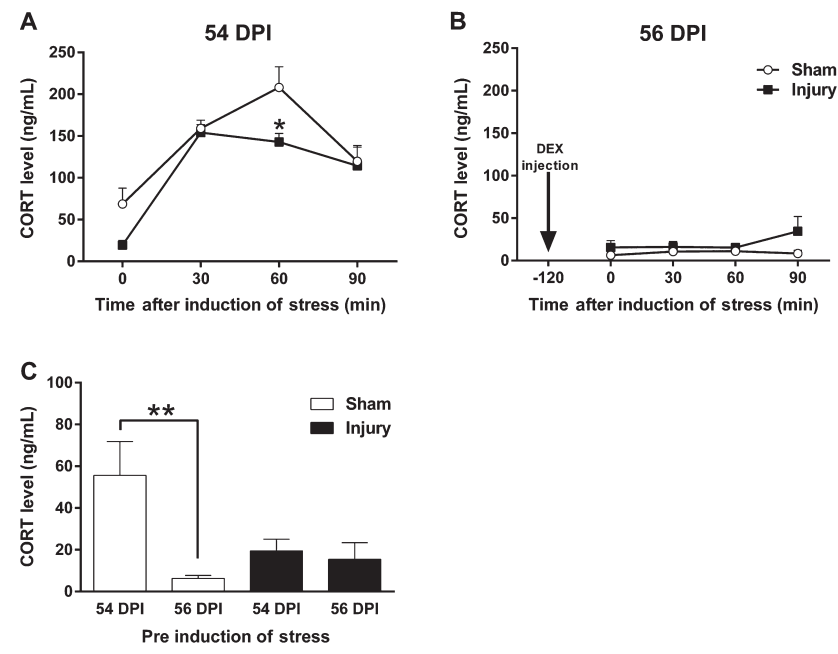

\section{Figure 2}

Diffuse TBI resulted in a blunted increase in CORT under stressed conditions, which was suppressed by DEX. (A) Restraint stress significantly increased plasma CORT across time in all rats, as expected. However, $60 \mathrm{~min}$ after stress onset, there was a blunted increase in CORT indicated by significantly lower CORT levels in brain-injured rats compared with shams. (Data presented as mean \pm S.E.M.; sham $n=7$, injured $n=6$, $* P<0.05)$. (B) Plasma levels of CORT were tested $2 \mathrm{~h}$ following dexamethasone (DEX) injections $(0.1 \mathrm{mg} / \mathrm{kg}$ ) at 56 DPI. DEX suppressed CORT levels similarly in both the brain-injured rats and uninjured shams under stressed conditions. (Data presented as mean \pm S.E.M.; sham $n=6$, injured $n=6$ ). (C) DEX treatment significantly reduced CORT levels in the uninjured shams, but not brain-injured rats, under resting conditions at 54 DPI compared with 56 DPI. (Data presented as mean \pm S.E.M.; $\operatorname{sham} n=6$, injury $n=6 ; * * P<0.01$ ). no change in CORT levels in brain-injured or sham rats as a function of time $(\mathrm{F}(3,30)=0.7555, P=0.5272$; Fig. $2 \mathrm{~B})$.

\section{DEX significantly reduced CORT levels under resting conditions in shams but not brain-injured rats}

A two-way ANOVA indicated a significant overall interaction between injured and sham rats at 54 DPI (prerestraint stress) compared with 56 DPI (following DEX prerestraint stress) $(F(1,9)=6.408, P=0.0322$; Fig. 2C). There was also a significant time effect between CORT levels measured at 54 DPI compared with 56 DPI $(\mathrm{F}(1,9)=5.980, \quad P=0.0370 ; \quad$ Fig. 2C). Sidak's multiple comparisons test indicated a significant difference between the sham CORT levels at 54 DPI compared with 56 DPI, but not between brain-injured 54 DPI compared with 56 DPI.

\section{Diffuse TBI resulted in a significantly greater change in body weight compared with uninjured shams}

Changes in body weight could represent injuryrelated metabolism and endocrine dysfunction. In the first 3 days after brain injury (from 0 to $3 \mathrm{DPI}$ ), TBI significantly decreased body weight compared with sham $(t(17)=3.394, P=0.0035$; Fig. 3A). In contrast, body weight increased from 3 to 58 DPI in all animals; however, brain-injured rats gained significantly greater amounts of body weight in comparison to sham $(\mathrm{t}(17)=5.066, P<0.0001$; Fig. 3B). Overall, brain injury led to acute decreases in body weight, which reversed and increased over time, such that weights of braininjured rats surpassed those of uninjured rats.
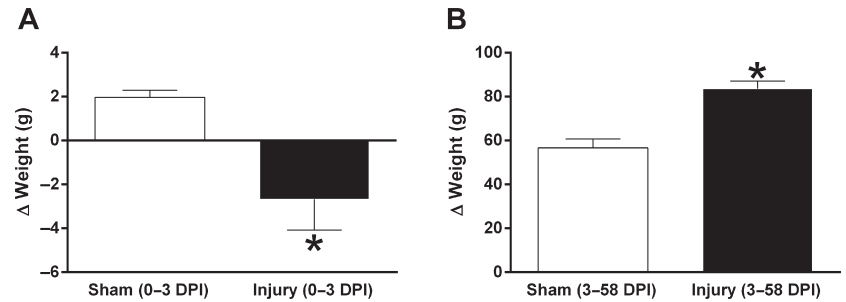

Figure 3

Diffuse TBI resulted in a significantly greater change in body weight compared with uninjured shams. (A) Brain injury significantly decreased body weight from 0 to 3 days postinjury compared with sham. (B) Changes in body weights from 3 to 58 days postinjury were significantly greater in brain-injured rats compared with sham. (Data presented as mean \pm S.E.M.; sham $n=10$, injury $n=9 ; * P<0.05$ ).

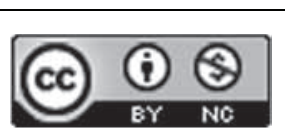

This work is licensed under a Creative Commons Attribution-NonCommercial 4.0 International License. 


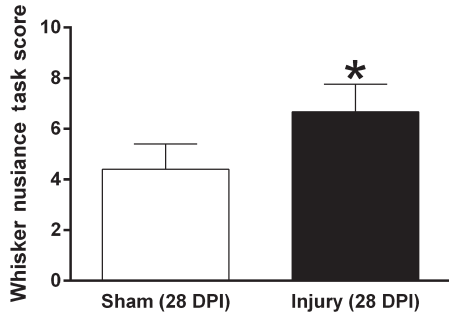

Figure 4

Sensory sensitivity was increased 28 days postinjury measured by the whisker nuisance task. Sensory sensitivity, assessed by the whisker nuisance task (WNT) at $28 \mathrm{DPI}$, was significantly increased compared with shams. These data support the late-onset of behavioral morbidity seen in this experimental model of diffuse TBI. (Data presented as mean \pm S.E.M.; sham $n=10$, injury $n=10 ; * P<0.05$ ).

\section{Sensory sensitivity was increased 28 DPI measured by the whisker nuisance task}

We have previously reported increased sensory sensitivity in rats following diffuse TBI (31), as an injury-induced morbidity. Diffuse brain injury resulted in a significantly increased sensory sensitivity, assessed by the whisker nuisance task (WNT) at $28 \mathrm{DPI}(\mathrm{U}(18)=27.00, P=0.0424$; Fig. 4).

\section{Diffuse TBI induced changes in neuron process complexity over time in the PVN}

TBI-induced damage to neurons in the PVN could impact HPE axis signaling involved with CORT regulation. The 3D traces (Fig. 5B) of parvocellular neurons (Fig. 5A)
A

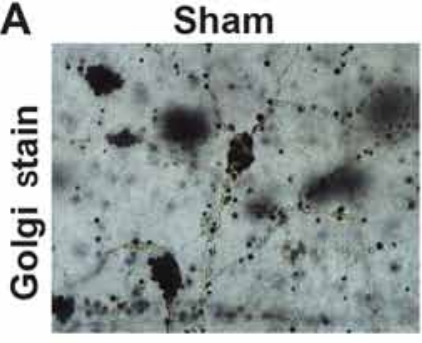

$1 \mathrm{DPI}$

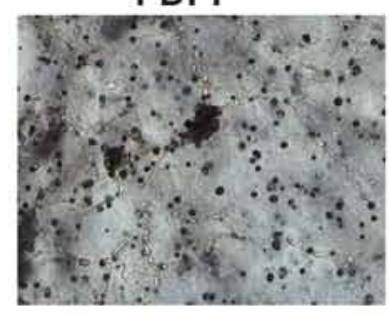

7 DPI
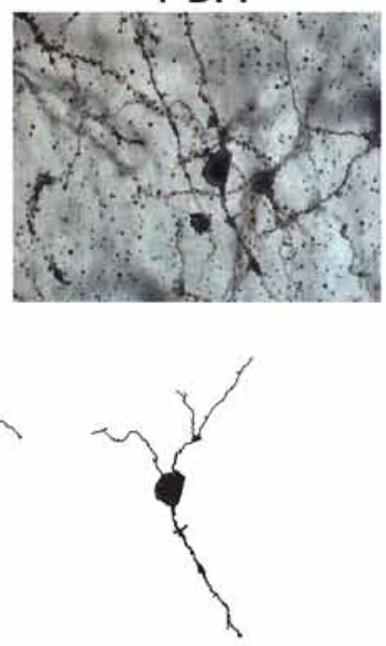

E
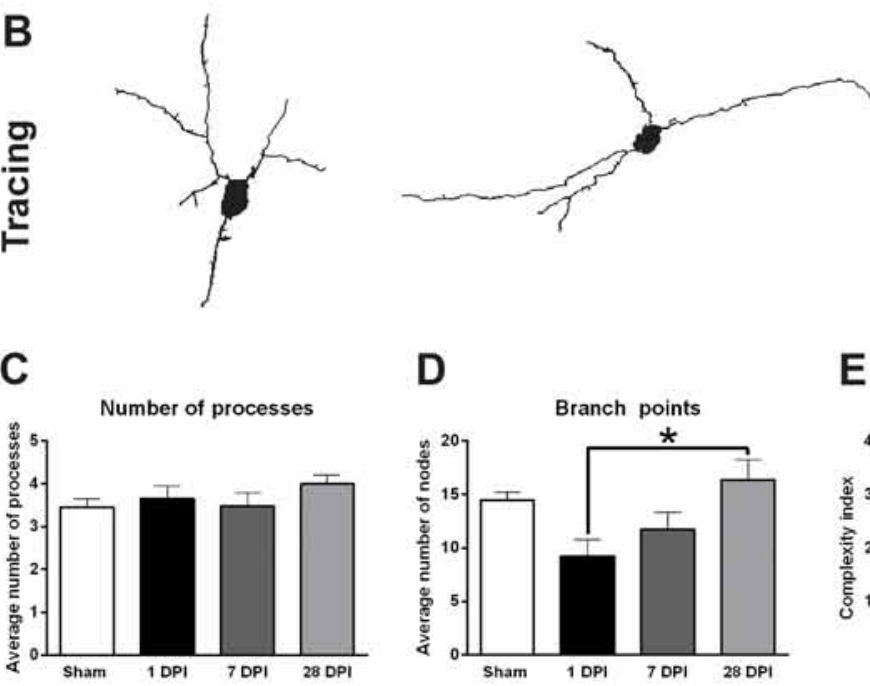

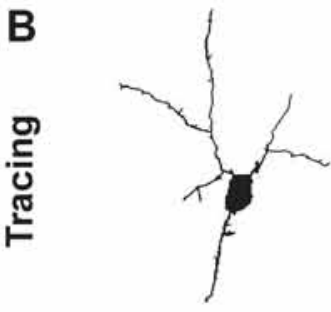

C $\begin{array}{llll}\text { Sham } 1 \mathrm{DPI} & 7 \mathrm{DPI} & 28 \mathrm{DPI} & \end{array}$

\section{D}

\section{DPI}
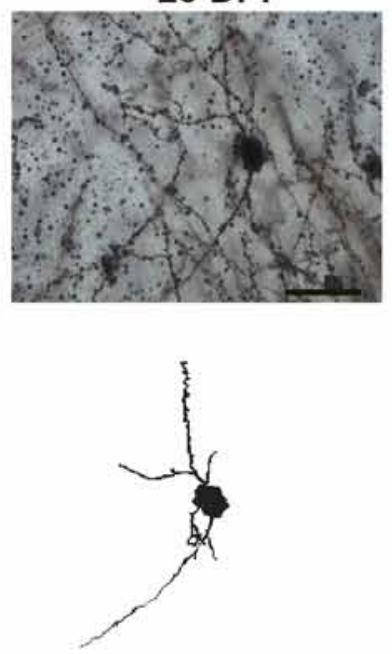

$\mathbf{F}$

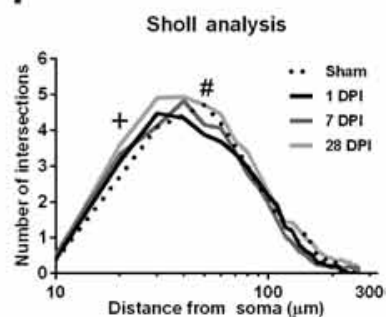

Figure 5

Golgi-stained neurons in the paraventricular nucleus display altered morphologies over the postinjury time course. (A) Representative neurons in the paraventricular nucleus of the hypothalamus are displayed at 40x magnification. (B) 3D reconstruction of neurons represented in panel A. (C) The number of processes were similar across all time points. (D) The number of branch points trended toward a decreased at 1 DPI, significantly increasing by $28 \mathrm{DPI}$. (E) The average complexity index significantly differed over time postinjury, with a significant increase at 28 DPI in comparison to 1 DPI and 7 DPI. (F) The Sholl analysis indicates that the complexity of the neurons changes as a function of days postinjury, with a greater number of intersections at $20 \mu \mathrm{m}$ from soma for $28 \mathrm{DPI}$ animals in comparison to sham (+) and significantly more intersections at $50 \mu \mathrm{m}$ for shams in comparison to $1 \mathrm{DPI}$ (\#) $\left({ }^{*} P<0.05\right)$. Bar graphs represent the mean \pm S.E.M.; scale bar $=50 \mu \mathrm{m} ;{ }^{*} P<0.05$.

http://www.endocrineconnections.org $\quad$ C 2016 The authors
DOI: $10.1530 /$ EC-16-0031


were quantified for average dendritic quantity, branch points and process complexity index at each time point following TBI (Fig. 5C, D and E). The number of dendrites was similar among treatments (sham, 1 DPI, 7 DPI, 28 DPI), with an average of 3-4 processes per neuron $(\mathrm{F}(3,11)=0.744, P=0.548)$. The number of branch points changed over days postinjury $(\mathrm{F}(3,11)=4.513, P=0.027)$, with 1 day approaching significance in comparison to sham $($ sham $=14.51 \pm 0.70 ; 1$ DPI $=9.23 \pm 1.6)$ and significantly increased at $28 \mathrm{DPI}$ in comparison to $1 \mathrm{DPI}$ (28 DPI $=16.40 \pm 1.8 ; \quad P<0.05$, Fig. 5D). The process complexity index also significantly changed over days postinjury $(\mathrm{F}(3,11)=9.897 ; P=0.002)$. Post hoc comparison indicated complexity differed between 1 DPI and 28 DPI as well as 7 DPI and 28 DPI $(P<0.05$; Fig. 5E).

The Sholl analysis represents the number of neuron axonal and dendritic branches at $10 \mu \mathrm{m}$ increments from the soma using concentric spheres (3D) to reflect process complexity (Fig. 5F). A two-way, repeated measures ANOVA revealed a significant effect of distance from the soma $(F(26,297)=191.0, P<0.0001)$, indicating the expected outcome that neuron process complexity changes as a function of distance from the soma. Importantly, the omnibus ANOVA showed a significant interaction for days postinjury as a function of the neuron processes' distance from the soma $(F(3,297)=9.781$, $P<0.0001)$. Post hoc analyses comparing differences at specific distances from the soma revealed significant differences between sham and 28 DPI at $20 \mu \mathrm{m}$ away from the soma, as well as between sham and 1 DPI at $50 \mu \mathrm{m}$ away from the soma. Together, the neuron morphology data suggest diffuse TBI resulted in altered neuronal process complexity over time postinjury in a brain region that regulates endocrine function.

\section{Paraventricular nucleus is absent of neuropathology after diffuse TBI}

The de Olmos aminocupric silver stain indicates neuropathology based on black-colored argyrophilic reaction products. There was no change in the amount of argyrophilic reaction product over days postinjury $(\mathrm{F}(3,8)=0.272 ; P=0.843$; Fig. 6$)$, with an average of $2-3 \%$ of argyrophilic reaction product accumulation measured across all time points, regardless of brain injury. These data indicate that neuropathology is not a significant contributor to changes in neuronal dendritic morphology.
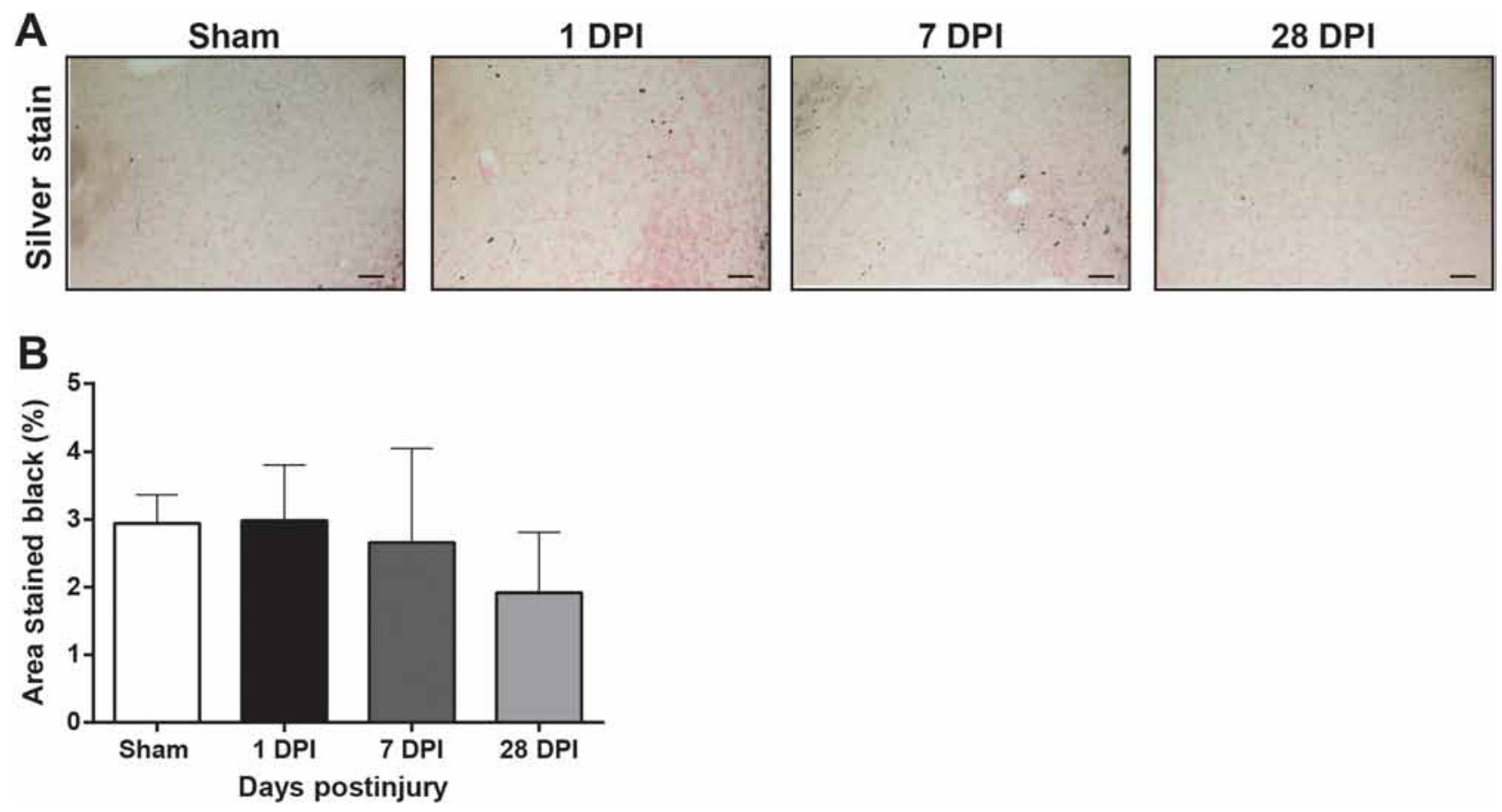

Figure 6

Absence of silver staining indicates no neuropathology in the paraventricular nucleus. (A) Representative photomicrographs are displayed at 20x magnification. (B) Quantification of argyrophilic reaction product accumulation shows no difference between sham, 1, 7 and 28 DPI. Data were analyzed using a one-way ANOVA. Bar graph represents mean \pm S.E.M.; scale bar $=50 \mu \mathrm{m}$.

\begin{tabular}{|lr}
\hline http://www.endocrineconnections.org & ○ 2016 The authors \\
DOI: $10.1530 /$ EC-16-0031 & Published by Bioscientifica Ltd.
\end{tabular}

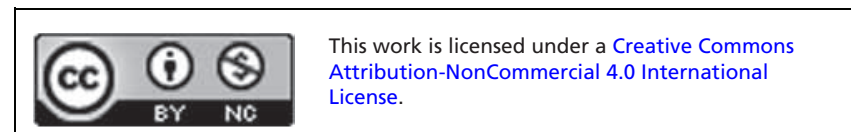



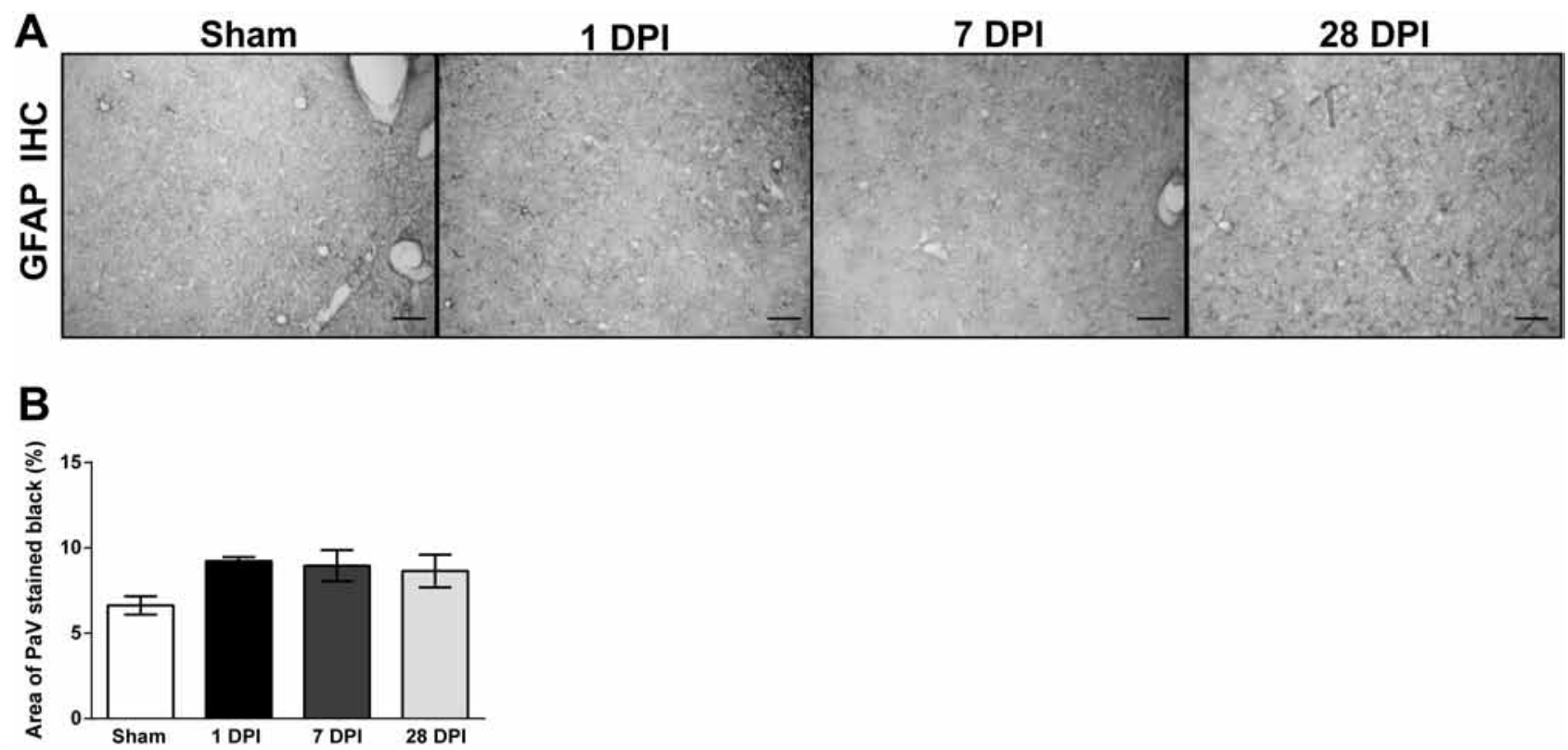

Figure 7

Absence of activated astrocytes in the paraventricular nucleus. (A) Representative photomicrographs of glial fibrillary acidic protein (GFAP) staining at 20x magnification in the PVN. (B) Quantification of GFAP staining was not different across time points (scale bar $=50 \mu m$; Bar graph represents mean \pm S.E.M.).

\section{Absence of activated astrocytes in the paraventricular nucleus}

The presence of activated astrocytes was assessed by immunohistochemistry for GFAP over time. At no time, postinjury was astrocytosis evident as a greater intensity or distribution of GFAP staining, indicative of activated astrocytes $\quad(\mathrm{F}(3,10)=2.719 ; \quad P=0.10)$. GFAP staining accounted for an average of $8.4 \%$ of the pixels across all time points, ranging from 6 to $11 \%$ between animals (Fig. 7). Therefore, diffuse TBI did not result in activated astrocytes in the paraventricular nucleus.

\section{Discussion}

Experimental diffuse TBI using midline fluid percussion in the rat showed evidence for CORT dysfunction at 54 days post-TBI. Plasma CORT levels were significantly decreased by approximately $60 \%$ at 54 DPI compared with baseline and uninjured shams. However, there were no significant changes in testosterone, indicating that the hypothalamic-pituitary-gonadal axis was intact at this time point. Diffuse TBI led to a blunted increase in CORT released under acute restraint stress, indicating an altered neuroendocrine stress response. After treatment with DEX, the HPE response to restraint stress was suppressed in both brain-injured and uninjured sham rats, indicating an intact feedback response. Diffuse TBI increased sensory sensitivity measured by the whisker nuisance task, and overall, brain-injured rats had a greater change in weight in comparison to sham at 58 DPI. The development of endocrine dysfunction, defined as decreased resting CORT levels and blunted CORT in response to stress, in brain-injured animals, was concomitant with altered neuron morphology in the paraventricular nucleus of the hypothalamus, which likely contributed to persistent dysfunction.

The data in this study indicate a significant decrease in plasma CORT, but not testosterone at a chronic time point following diffuse TBI in the rat. Both clinical and experimental data suggest that TBI induces long-term hypopituitarism, with measureable deficiencies in both testosterone and cortisol. Prospective clinical screening for hypopituitarism in patients with brain injuries showed that the most common endocrine disturbance following brain injury was a decrease in testosterone, measured in $40.7 \%$ of all men, with low cortisol also being observed (56). Similarly, a retrospective clinical study identified hypopituitarism in nearly $70 \%$ of men following TBI, with $66 \%$ presenting low testosterone levels within the first 6 months of injury (57), but cortisol levels were not reported. In the current study, testosterone was not significantly lower at 54 DPI; however, CORT was significantly decreased compared with uninjured sham http://www.endocrineconnections.org DOI: 10.1530/EC-16-0031 (c) 2016 The authors Published by Bioscientifica Ltd.
This work is licensed under a Creative Commons Attribution-NonCommercial 4.0 International License. 
levels at 54 DPI. It is possible that testosterone levels had decreased postinjury, but recovered to baseline levels by 54 DPI in the current experimental model of TBI; future studies on serial plasma measurements may determine the course of testosterone in the brain-injured rat.

The chronic decrease in CORT measured in this study parallels both clinical and experimental data suggesting that TBI leads to chronic deficiencies in cortisol (56). Deficiencies in CORT include symptoms such as weakness, dizziness, inability to cope with stress, nausea and vomiting $(58,59)$, which parallel and overlap postconcussive syndrome symptoms. The difficulty in a differential diagnosis between TBI and endocrine dysfunction may reflect the broad epidemiology reports in the literature. Brain injuryinduced hormone deficiencies are associated with increased morbidity and poor recovery (60). While there are no data to support hormone replacement reducing mortality following TBI, early detection and supplementation can improve functional outcome and quality of life in TBI survivors (60).

The HPE axis is activated by stressful events that disrupt homeostasis, such as the restraint stress used in the present study $(33,61)$. The stress response starts with the activation of parvocellular secretory neurons of the paraventricular nucleus in the hypothalamus. These neurons release $\mathrm{CRH}$ into the superior hypophyseal portal vein, which stimulates the release of ACTH from the anterior pituitary, and finally the release of CORT from the adrenal gland into circulation. We found that restraint stress increased plasma CORT levels in all rats. However, $60 \mathrm{~min}$ after stress onset, plasma CORT levels were significantly lower in brain-injured rats compared with uninjured shams. In lateral FPI, a model of mixed focal and diffuse injury, CORT levels were significantly lower postrestraint at 7 DPI; however, as a percent increase from basal CORT levels, FPI increased CORT in response to stress (35). Restraint stress following controlled cortical impact (CCI) focal brain injury showed that HPE responses were attenuated at 7 and 21 DPI compared with sham (32). Similarly, 4 weeks after CCI in rats, plasma CORT response to $30 \mathrm{~min}$ of restraint stress was significantly blunted in brain-injured rats compared with shams (34). CORT response measured post-TBI can identify neuroendocrine dysfunction; however, acute restraint stress reveals effects of TBI on stress coping behavior. Our studies are the first to explore chronic endocrine function following diffuse brain injury using mFPI, and further studies are necessary to evaluate short-term changes to the neuroendocrine stress response following injury.
A potential mechanism of dysregulation of the endocrine system can be attributed to the injury-induced effects on the sensitivity of negative feedback systems to glucocorticoids following TBI in the rat (33). Provocative testing, as accomplished in the rat by dexamethasone (DEX) administration followed by restraint stress, can be used to uncover damage to the HPE axis $(33,35)$. The HPE axis is the central stress response system and works through a negative-feedback control. For this study, we administered the synthetic glucocorticoid analog, DEX, which we hypothesized would facilitate the negative feedback control of the HPE axis as demonstrated by lowered plasma CORT levels. As expected, restraint stress following DEX injections resulted in decreased CORT levels of both brain-injured and uninjured shams, without difference between groups. Similarly, DEX has been shown to suppress plasma CORT in rats following lateral FPI (35). DEX has also been shown to suppress CORT at 7 and 35 DPI following CCI in rats (33). The current study demonstrated an intact negative feedback system in which DEX suppressed CORT levels in braininjured rats following stress. Since the HPE axis is sensitive to feedback, it is possible that the present design saturated the system and occluded differences between groups. While the dose used in this study was guided by a literature search of DEX used in rats post-TBI $(33,43,44$, 45), a lower dose of DEX may uncover subtle alterations and injury-induced deficits in CORT feedback.

While DEX suppressed CORT following restraint stress, it should be noted that the prestress baseline CORT levels measured at 56 DPI were lower in brain-injured rats compared with uninjured sham rats compared with their baseline CORT levels measured at 54 DPI. There was a significant decrease in baseline levels of plasma CORT prestress in the uninjured shams at 56 DPI compared with their baseline plasma CORT at 54 DPI, indicating that DEX decreased CORT and continued to suppress CORT production under stressed conditions. Contrarily, there was no difference in baseline levels of plasma CORT prestress at 56 DPI in brain-injured rats following DEX administration compared with their baseline plasma CORT at 54 DPI. Diffuse TBI led to decreased CORT levels by 56 DPI, such that negative feedback by DEX administration could not further suppress CORT levels.

The production and release of CORT is regulated by ACTH which is produced by the anterior pituitary. The currently observed decrease in CORT may be attributed to decreased ACTH production due to damage to the anterior pituitary. In the current study, brain-injured rats have increased weight gain concomitantly with chronic

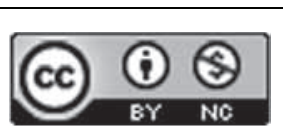

This work is licensed under a Creative Commons Attribution-NonCommercial 4.0 International License. 
CORT deficiencies. Damage to the anterior pituitary could not only decrease ACTH and CORT, but can contribute to decreased thyroid-stimulating hormone (TSH) production. TSH is a glycoprotein hormone secreted in the anterior pituitary and is responsible for stimulating metabolism of tissue throughout the body. Decreased TSH, referred to as hypothyroidism, results in weight gain, and is a common symptom of TBI survivors $(15,62,63,64)$. In a prospective study assessing functional outcome in patients with TBI, 32\% presented hypothyroidism at 3 months postinjury (62). This parallels a longitudinal follow-up of adults with TBI showed over a median period of 38 months, $42 \%$ of TBI patients had gained weight (65). In the current study, brain-injured rats lost weight immediately following injury, but had an overall significant increase in weight at 58 DPI compared with uninjured shams. It is possible that damage to the anterior pituitary resulted in decreased TSH levels and contributed to this weight gain. Growth hormone deficiency has also been associated with weight gain; however, weight redistribution due to altered body composition (increased fat and decreased lean body mass) more accurately describes the long-term effects $(66,67)$. Alternatively, decreased activity or increased food intake could have contributed to weight gain. Limitations of the current study include group housing which prevented the assessment of metabolic parameters including food and water intake. Further studies are needed to measure metabolic parameters and levels of TSH and GH, and examine injury-induced pathology to the pituitary to determine if the chronic weight gain is a symptom of neuroendocrine dysfunction following TBI.

While there is no overt cell death in our injury model, our group has accumulated evidence for neuronal circuit dismantling leading to maladaptive circuit reorganization in the diffuse-injured brain $(31,38,39)$. These alterations in neuronal and circuit function can likely be attributed to reparative responses to the brain injury. Consequences of circuit disruption following diffuse brain injury include increased sensory sensitivity to manual stimulation of the facial whiskers $(31,39,41)$. Additionally, this sensory stimulation resulting from the whisker nuisance task significantly elevated plasma levels of CORT in brain-injured animals compared with sham animals (31). The once non-noxious stimulus of whisker stimulation now induces a stress response in the diffuse braininjured animal $(31,68)$. In the current study, we used the whisker nuisance task as a behavioral outcome measure confirming late-onset injury-induced sensory sensitivity. Brain injury resulted in a significantly increased sensory sensitivity at $28 \mathrm{DPI}$. The same animals went on to demonstrate decreased baseline CORT at 54 DPI, indicating that this late-onset of behavioral morbidity following diffuse TBI in the rat may be related to chronic endocrine dysfunction. Changes in neuronal and circuit function can affect hypothalamic and pituitary hormonal release. Chronic decreased CORT and blunted CORT release in response to stress are indicative of intact pituitary and hypothalamic neuronal connections with possible hypothalamic disruptions in circuitry. Examination of neuron process complexity of neurons within the PVN was used to determine structural changes that could contribute to the observed functional change. In the current study, neuron process complexity in the medial PVN of the hypothalamus changed over 1 month postinjury. These changes were most evident between 1 and 28 DPI, with the support that neuron process complexity was most highly impacted within $20 \mu \mathrm{m}$ of the soma. The total number of processes over time did not change, supporting that the changes were due to increased number of branches per process, rather than new or more processes.

The trend for fewer branch points at 1 DPI with more at 28 DPI could result from direct damage to the processes from the mechanical forces of the brain injury, followed by regenerative attempts to repair neuronal architecture at 7 and 28 DPI. However, silver staining indicated that there were no major changes in neuron pathology at 1, 7 or 28 DPI. Changes in PVN process complexity can also be mediated by neurotrophic factors produced from activated astrocytes; however, GFAP staining or astrocyte morphology do not support this mechanism either. Alternatively, changes in input from brain regions that regulate the PVN and release of CRH via feedback through glucocorticoid and mineralocorticoid receptors (hippocampus, amygdala, prefrontal cortex and midbrain) could also contribute to compensatory changes in neuron complexity $(69,70)$. There is evidence of early and persistent dendritic hypertrophy of pyramidal and spiny stellate neurons in the basolateral amygdala from the same cohort of animals used for Golgi staining in this study (71). In summary, the specific mechanisms by which brain injury can change neuron dendritic complexity within the PVN remain to be determined. However, while these structural changes are subtle, their contribution to chronic neuroendocrine disruption should be taken into consideration in regards to hormone regulation.

This brain region has not been explored for a homeostatic neuronal response in the wake of brain injury. While the data are indicative of injury-induced

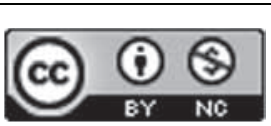


changes, several limitations must be considered. First, the amount of plasma that could be collected which restricted the number of hormones (e.g. growth hormone, ACTH, CRH) that could be evaluated. Serial blood draws were necessary for the repeated sampling used during restraint stress and the DEX challenge. While the current study examined the hypothalamic PVN neurons, further evaluation of the pituitary itself and input regions (e.g. amygdala) is encouraged. Lastly, the current study did not test for hypogonadism. Further investigation of bound testosterone, free testosterone, sex hormone binding globulin and albumin is necessary to fully elucidate the role of TBI on testosterone production.

A limitation of Golgi staining is that, despite being able to differentiate cells by morphology, specific cell type and connections cannot be determined. The identification of parvocellular neurons based on morphology and location in the medial paraventricular hypothalamic nucleus is not fail-proof $(47,48,49,50)$. Immunohistochemical labeling for CRH would aid the identification of parvocellular neurons specifically; however, stress conditions may lead to other cell types (i.e. magnocellular neurons) expressing CRH (49). Still, the morphology of neurons represented in the data set is typical of neurons in the medial paraventricular nucleus $(47,72,73,74,75)$.

\section{Conclusion}

Diffuse TBI resulted in chronic CORT dysfunction including chronic decrease in plasma CORT, blunted CORT response to restraint stress, increased sensory sensitivity to non-noxious stimuli, and increased weight gain. The HPE response to stress was suppressed by DEX in both brain-injured and uninjured shams. Diffuse TBI altered neuron morphology in the PVN of the hypothalamus over time postinjury. This suggests injury to the hypothalamus associated with chronic changes in morphology to the neurons contributes to neuroendocrine dysfunction. Additional studies are necessary to examine pathological damage to the hypothalamus and the pituitary following a single diffuse brain injury.

\section{Declaration of interest}

The authors declare that there is no conflict of interest that could be perceived as prejudicing the impartiality of the research reported.

\section{Funding}

Research reported in this manuscript was supported, in part, by Phoenix VA Health Care System, Arizona Biomedical Research Commission as made available through the Arizona Department of Health Services under award number ADHS14-00003606, National Institute of Neurological Disorders and Stroke of the National Institutes of Health under award number R01 NS-065052, Phoenix Children's Hospital Leadership Circle and Phoenix Children's Hospital Mission Support Funds. Dr Rowe was supported by the Bisgrove Scholar award funded by Science Foundation Arizona.

\section{Acknowledgments}

The contents of this article do not represent the views of the Department of Veterans Affairs or the US Government. The authors would like to thank Cheryl D Conrad, PhD, for valuable feedback on the manuscript, and Wuqiong Ma, Daniel Griffiths, Megan Evilsizor and Taylor Colburn for their technical assistance and contribution to the studies.

\section{References}

1 Morissette SB, Woodward M, Kimbrel NA, Meyer EC, Kruse MI Dolan S \& Gulliver SB. Deployment-related TBI, persistent postconcussive symptoms, PTSD, and depression in OEF/OIF veterans. Rehabilitation Psychology 201156 340-350. (doi:10.1037/a0025462)

2 Masel BE \& DeWitt DS. Traumatic brain injury: a disease process, not an event. Journal of Neurotrauma 201027 1529-1540. (doi:10.1089/ neu.2010.1358)

3 Werner C \& Engelhard K. Pathophysiology of traumatic brain injury. British Journal of Anaesthesia 200799 4-9. (doi:10.1093/bja/aem131)

4 Munoz A, Liu XB \& Jones EG. Development of metabotropic glutamate receptors from trigeminal nuclei to barrel cortex in postnatal mouse. Journal of Comparative Neurology 1999409 549-566. (doi:10.1002/ (SICI)1096-9861(19990712)409:4<549::AID-CNE3>3.3.CO;2-9)

5 Tanriverdi F \& Kelestimur F. Neuroendocrine disturbances after brain damage: an important and often undiagnosed disorder. Journal of Clinical Medicine 20154 847-857. (doi:10.3390/jcm4050847)

6 Gasco V, Prodam F, Pagano L, Grottoli S, Belcastro S, Marzullo P, Beccuti G, Ghigo E \& Aimaretti G. Hypopituitarism following brain injury: when does it occur and how best to test? Pituitary 201215 20-24. (doi:10.1007/s11102-010-0235-6)

7 Shaw NA. The neurophysiology of concussion. Progress in Neurobiology 200267 281-344. (doi:10.1016/S0301-0082(02)00018-7)

8 McAllister TW. Neuropsychiatric sequelae of head injuries. Psychiatric Clinics of North America 199215 395-413.

9 Glynn N \& Agha A. Which patient requires neuroendocrine assessment following traumatic brain injury, when and how? Clinical Endocrinology 201378 17-20. (doi:10.1111/cen.12010)

10 Wilkinson CW, Pagulayan KF, Petrie EC, Mayer CL, Colasurdo EA, Shofer JB, Hart KL, Hoff D, Tarabochia MA \& Peskind ER. High prevalence of chronic pituitary and target-organ hormone abnormalities after blast-related mild traumatic brain injury. Frontiers in Neurology 20123 11. (doi:10.3389/fneur.2012.00011)

11 Berg C, Oeffner A, Schumm-Draeger PM, Badorrek F, Brabant G, Gerbert B, Bornstein S, Zimmermann A, Weber M, Broecker-Preuss M, et al. Prevalence of anterior pituitary dysfunction in patients following traumatic brain injury in a German multi-centre screening program. Experimental and Clinical Endocrinology \& Diabetes 2009118 139-144. (doi:10.1055/s-0029-1225611)

12 Kelly DF, McArthur DL, Levin H, Swimmer S, Dusick JR, Cohan P, Wang C \& Swerdloff R. Neurobehavioral and quality of life changes associated with growth hormone insufficiency after complicated mild, moderate, or severe traumatic brain injury. Journal of Neurotrauma 200623 928-942. (doi:10.1089/neu.2006.23.928)

13 Zaben M, El Ghoul W \& Belli A. Post-traumatic head injury pituitary dysfunction. Disability and Rehabilitation 201335 522-525. (doi:10.31 09/09638288.2012.697252)

14 Webb NE, Little B, Loupee-Wilson S \& Power EM. Traumatic brain injury and neuro-endocrine disruption: medical and psychosocial

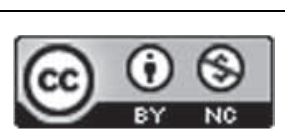

This work is licensed under a Creative Commons Attribution-NonCommercial 4.0 International License. 
rehabilitation. NeuroRehabilitation 201434 625-636. (doi:10.3233/ NRE-141074)

15 Nemes O, Kovacs N, Czeiter E, Kenyeres P, Tarjanyi Z, Bajnok L, Buki A, Doczi T \& Mezosi E. Predictors of post-traumatic pituitary failure during long-term follow-up. Hormones 201514 383-391. (doi:10.14310/horm.2002.1564)

16 Bavisetty S, Bavisetty S, McArthur DL, Dusick JR, Wang C, Cohan P, Boscardin WJ, Swerdloff R, Levin H, Chang DJ, Muizelaar JP, et al. Chronic hypopituitarism after traumatic brain injury: risk assessment and relationship to outcome. Neurosurgery 200862 1080-1093. (doi:10.1227/01.neu.0000325870.60129.6a)

17 Agha A, Rogers B, Sherlock M, O'Kelly P, Tormey W, Phillips J \& Thompson CJ. Anterior pituitary dysfunction in survivors of traumatic brain injury. Journal of Clinical Endocrinology and Metabolism 200489 4929-4936. (doi:10.1210/jc.2004-0511)

18 Kleindienst A, Hannon MJ, Buchfelder M \& Verbalis JG. Hyponatremia in neurotrauma: the role of vasopressin. Journal of Neurotrauma 201633 615-624. (doi:10.1089/neu.2015.3981)

19 Cole CD, Gottfried ON, Liu JK \& Couldwell WT. Hyponatremia in the neurosurgical patient: diagnosis and management. Neurosurgical Focus 200416 E9.

20 Richmond E \& Rogol AD. Traumatic brain injury: endocrine consequences in children and adults. Endocrine 2014 45 3-8. (doi:10.1007/s12020-013-0049-1)

21 Popovic V, Aimaretti G, Casanueva FF \& Ghigo E. Hypopituitarism following traumatic brain injury. Growth Hormone \& IGF Research 2005 15 177-184. (doi:10.1016/j.ghir.2005.02.003)

22 Casanueva FF, Ghigo E \& Popovic V. Hypopituitarism following traumatic brain injury (TBI): a guideline decalogue. Journal of Endocrinological Investigation 200427 793-795. (doi:10.1007/BF03347526)

23 Dubourg J \& Messerer M. Sports-related chronic repetitive head trauma as a cause of pituitary dysfunction. Neurosurgical Focus 2011 31 E2. (doi:10.3171/2011.8.focus11182)

24 VandeVord PJ, Sajja VS, Ereifej E, Hermundstad A, Mao S \& Hadden TJ. Chronic hormonal imbalance and adipose redistribution is associated with hypothalamic neuropathology following blast exposure. Journal of Neurotrauma 201633 82-88. (doi:10.1089/ neu.2014.3786)

25 Baxter D, Sharp DJ, Feeney C, Papadopoulou D, Ham TE, Jilka S, Hellyer PJ, Patel MC, Bennett AN, Mistlin A, et al. Pituitary dysfunction after blast traumatic brain injury: the UK BIOSAP study. Annals of Neurology 201374 527-536. (doi:10.1002/ana.23958)

26 Tanriverdi F \& Kelestimur F. Pituitary dysfunction following traumatic brain injury: clinical perspectives. Neuropsychiatric Disease and Treatment 201511 1835-1843. (doi:10.2147/ndt.s65814)

27 Tanriverdi F, Schneider HJ, Aimaretti G, Masel BE, Casanueva FF \&Kelestimur F. Pituitary dysfunction after traumatic brain injury: a clinical and pathophysiological approach. Endocrine Reviews 201536 305-342. (doi:10.1210/er.2014-1065)

28 Thomas TC, Colburn TA, Korp K, Khodadad A \& Lifshitz J. Translational considerations for behavioral impairment and rehabilitation strategies after diffuse traumatic brain injury. In Brain Neurotrauma: Molecular, Neuropsychological, and Rehabilitation Aspects, Ed FH Kobeissy. Boca Raton, FL, USA: CRC Press/Taylor \& Francis, 2015.

29 Popovic V, Pekic S, Pavlovic D, Maric N, Jasovic-Gasic M, Djurovic B, Medic Stojanoska M, Zivkovic V, Stojanovic M, Doknic M, et al. Hypopituitarism as a consequence of traumatic brain injury (TBI) and its possible relation with cognitive disabilities and mental distress. Journal of Endocrinological Investigation 200427 1048-1054. (doi:10.1007/bf03345308)

30 Cernak I, Savic VJ, Lazarov A, Joksimovic M \& Markovic S. Neuroendocrine responses following graded traumatic brain injury in male adults. Brain Injury 199913 1005-1015. (doi:10.1080/026990599121016)

31 McNamara KC, Lisembee AM \& Lifshitz J. The whisker nuisance task identifies a late-onset, persistent sensory sensitivity in diffuse brain-injured rats. Journal of Neurotrauma 201027 695-706. (doi:10.1089/neu.2009.1237)

32 Taylor AN, Rahman SU, Sanders NC, Tio DL, Prolo P \& Sutton RL. Injury severity differentially affects short- and long-term neuroendocrine outcomes of traumatic brain injury. Journal of Neurotrauma 200825 311-323. (doi:10.1089/neu.2007.0486)

33 Taylor AN, Rahman SU, Tio DL, Gardner SM, Kim CJ \& Sutton RL. Injury severity differentially alters sensitivity to dexamethasone after traumatic brain injury. Journal of Neurotrauma 201027 1081-1089. (doi:10.1089/neu.2009.1252)

34 Taylor AN, Rahman SU, Tio DL, Sanders MJ, Bando JK, Truong AH $\&$ Prolo P. Lasting neuroendocrine-immune effects of traumatic brain injury in rats. Journal of Neurotrauma 200623 1802-1813. (doi:10.1089/neu.2006.23.1802)

35 Griesbach GS, Hovda DA, Tio DL \& Taylor AN. Heightening of the stress response during the first weeks after a mild traumatic brain injury. Neuroscience 2011178 147-158. (doi:10.1016/j.neuroscience.2011.01.028)

36 Lifshitz J. Fluid percussion injury model. In Animal Models of Acute Neurological Injuries, pp 369-384. Eds J Chen, XM Xu, ZC Xu \& JH Zhang. Totowa, NJ, USA: Humana Press, 2007.

37 Ziebell JM, Taylor SE, Cao T, Harrison JL \& Lifshitz J. Rod microglia: elongation, alignment, and coupling to form trains across the somatosensory cortex after experimental diffuse brain injury. Journal of Neuroinflammation 20129 247. (doi:10.1186/1742-2094-9-247)

38 Cao T, Thomas TC, Ziebell JM, Pauly JR \& Lifshitz J. Morphological and genetic activation of microglia after diffuse traumatic brain injury in the rat. Neuroscience 2012225 65-75. (doi:10.1016/j. neuroscience.2012.08.058)

39 Thomas TC, Hinzman JM, Gerhardt GA \& Lifshitz J. Hypersensitive glutamate signaling correlates with the development of late-onset behavioral morbidity in diffuse brain-injured circuitry. Journal of Neurotrauma 201229 187-200. (doi:10.1089/neu.2011.2091)

40 Hosseini AH \& Lifshitz J. Brain injury forces of moderate magnitude elicit the fencing response. Medicine and Science in Sports and Exercise 200941 1687-1697. (doi:10.1249/mss.0b013e31819fcd1b)

41 Learoyd AE \& Lifshitz J. Comparison of rat sensory behavioral tasks to detect somatosensory morbidity after diffuse brain-injury. Behavioural Brain Research 2012226 197-204. (doi:10.1016/j.bbr.2011.09.016)

42 Gomez F, De Kloet ER \& Armario A. Glucocorticoid negative feedback on the HPA axis in five inbred rat strains. American Journal of Physiology 1998274 R420-R427.

43 Xu FF, Sun S, Ho AS, Lee D, Kiang KM, Zhang XQ, Wang AM, Wu EX, Lui WM, Liu BY, et al. Effects of progesterone vs. dexamethasone on brain oedema and inflammatory responses following experimental brain resection. Brain Injury 201428 1594-1601. (doi:10.3109/ 02699052.2014.943289)

44 Zhu H, Zhao Z, Zhou Y, Chen X, Li Y, Liu X, Lu H, Zhang Y \& Zhang J. High-dose glucocorticoid aggravates TBI-associated corticosteroid insufficiency by inducing hypothalamic neuronal apoptosis. Brain Research 2013 1541 69-80. (doi:10.1016/j.brainres.2013.10.002)

45 Chen X, Lin YP, Wang D \& Zhang JN. Dexamethasone exacerbates spatial acquisition deficits after traumatic brain injury in rats. Neurological Research 201032 1097-1102. (doi:10.1179/0161641 10x12681290831162)

46 Pillai AG, de Jong D, Kanatsou S, Krugers H, Knapman A, Heinzmann JM, Holsboer F, Landgraf R, Joëls M \& Touma C. Dendritic morphology of hippocampal and amygdalar neurons in adolescent mice is resilient to genetic differences in stress reactivity. PLOS ONE 20127 e38971. (doi:10.1371/journal.pone.0038971)

47 Wamsteeker Cusulin JI, Füzesi T, Watts AG \& Bains JS. Characterization of corticotropin-releasing hormone neurons in the paraventricular nucleus of the hypothalamus of Crh-IRES-Cre mutant mice. PLoS ONE 20138 e64943. (doi:10.1371/journal. pone.0064943)

48 Rho JH \& Swanson LW. A morphometric analysis of functionally defined subpopulations of neurons in the paraventricular nucleus

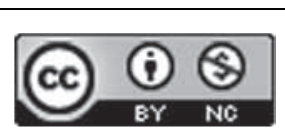

This work is licensed under a Creative Commons Attribution-NonCommercial 4.0 International License. 
of the rat with observations on the effects of colchicine. Journal of Neuroscience 19899 1375-1388.

49 Dabrowska J, Hazra R, Guo JD, Dewitt S \& Rainnie DG. Central CRF neurons are not created equal: phenotypic differences in CRFcontaining neurons of the rat paraventricular hypothalamus and the bed nucleus of the stria terminalis. Frontiers in Neuroscience 20137 156. (doi:10.3389/fnins.2013.00156)

50 Tasker JG \& Dudek FE. Electrophysiological properties of neurones in the region of the paraventricular nucleus in slices of rat hypothalamus. Journal of Physiology 1991434 271-293. (doi:10.1113/ jphysiol.1991.sp018469)

51 van den Pol AN. The magnocellular and parvocellular paraventricular nucleus of rat: intrinsic organization. Journal of Comparative Neurology 1982206 317-345. (doi:10.1002/cne.902060402)

52 Switzer RC 3rd. Application of silver degeneration stains for neurotoxicity testing. Toxicologic Pathology 200028 70-83. (doi:10.1177/019262330002800109)

53 de Olmos JS, Beltramino CA \& de Olmos de LS. Use of an aminocupric-silver technique for the detection of early and semiacute neuronal degeneration caused by neurotoxicants, hypoxia, and physical trauma. Neurotoxicology and Teratology 199416 545-561. (doi:10.1016/0892-0362(94)90033-7)

54 Mikics E, Baranyi J \& Haller J. Rats exposed to traumatic stress bury unfamiliar objects - a novel measure of hyper-vigilance in PTSD models? Physiology \& Behavior 200894 341-348. (doi:10.1016/ j.physbeh.2008.01.023)

55 Lifshitz J \& Lisembee AM. Neurodegeneration in the somatosensory cortex after experimental diffuse brain injury. Brain Structure and Function 2012217 49-61. (doi:10.1007/s00429-011-0323-z)

56 Kopczak A, Kilimann I, von Rosen F, Krewer C, Schneider HJ, Stalla GK \& Schneider M. Screening for hypopituitarism in 509 patients with traumatic brain injury or subarachnoid hemorrhage. Journal of Neurotrauma 201431 99-107. (doi:10.1089/neu.2013.3002)

57 Rosario ER, Aqeel R, Brown MA, Sanchez G, Moore C \& Patterson D. Hypothalamic-pituitary dysfunction following traumatic brain injury affects functional improvement during acute inpatient rehabilitation. Journal of Head Trauma Rehabilitation 201328 390-396. (doi:10.1097/ HTR.0b013e318250eac6)

58 Schneider HJ, Aimaretti G, Kreitschmann-Andermahr I, Stalla GK \& Ghigo E. Hypopituitarism. Lancet 2007369 1461-1470. (doi:10.1016/ S0140-6736(07)60673-4)

59 Schneider HJ, Corneli G, Kreitschman-Andermahr I, Rovere S, Bellone S, Bona G, Ghigo E \& Aimaretti G. Traumatic brain injury and hypopituitarism in children and adolescents: is the problem underestimated? Pediatric Endocrinology Reviews 20074 205-209.

60 Javed Z, Qamar U \& Sathyapalan T. Pituitary and/or hypothalamic dysfunction following moderate to severe traumatic brain injury: current perspectives. Indian Journal of Endocrinology and Metabolism 201519 753-763. (doi:10.4103/2230-8210.167561)

61 Dziedzic N, Dziedzic N, Ho A, Adabi B, Foilb AR \& Romeo RD. Shifts in hormonal stress reactivity during adolescence are not associated with changes in glucocorticoid receptor levels in the brain and pituitary of male rats. Developmental Neuroscience 201436 261-268. (doi:10.1159/000362873)

62 Marina D, Klose M, Nordenbo A, Liebach A \& Feldt-Rasmussen U. Early endocrine alterations reflect prolonged stress and relate to 1-year functional outcome in patients with severe brain injury. European Journal of Endocrinology 2015172 813-822. (doi:10.1530/ EJE-14-1152)

63 Kokshoorn NE, Wassenaar MJ, Biermasz NR, Roelfsema F, Smit JW, Romijn JA \& Pereira AM. Hypopituitarism following traumatic brain injury: prevalence is affected by the use of different dynamic tests and different normal values. European Journal of Endocrinology 2010162 11-18. (doi:10.1530/EJE-09-0601)

64 Powner DJ, Boccalandro C, Alp MS \& Vollmer DG. Endocrine failure after traumatic brain injury in adults. Neurocritical Care 20065 61-70. (doi:10.1385/ncc:5:1:61)

65 Crenn P, Hamchaoui S, Bourget-Massari A, Hanachi M, Melchior JC $\&$ Azouvi P. Changes in weight after traumatic brain injury in adult patients: a longitudinal study. Clinical Nutrition 201433 348-353. (doi:10.1016/j.clnu.2013.06.003)

66 Newman CB, Carmichael JD \& Kleinberg DL. Effects of low dose versus high dose human growth hormone on body composition and lipids in adults with GH deficiency: a meta-analysis of placebo-controlled randomized trials. Pituitary 201518 297-305. (doi:10.1007/s11102-014-0571-z)

67 Mangiola A, Vigo V, Anile C, De Bonis P, Marziali G \& Lofrese G. Role and importance of IGF-1 in traumatic brain injuries. BioMed Research International 20152015 736104. (doi:10.1155/2015/736104)

68 Lafrenaye AD, Krahe TE \& Povlishock JT. Moderately elevated intracranial pressure after diffuse traumatic brain injury is associated with exacerbated neuronal pathology and behavioral morbidity in the rat. Journal of Cerebral Blood Flow \& Metabolism 201434 1628-1636. (doi:10.1038/jcbfm.2014.122)

69 Tripodi M, Evers JF, Mauss A, Bate M, Landgraf M. Structural homeostasis: compensatory adjustments of dendritic arbor geometry in response to variations of synaptic input. PLoS Biology 20086 e260. (doi:10.1371/journal.pbio.0060260)

70 Yin J, Yuan Q. Structural homeostasis in the nervous system: a balancing act for wiring plasticity and stability. Frontiers in Cellular Neuroscience 20148 439. (doi:10.3389/fncel.2014.00439)

71 Hoffman AN, Paode PR, May HG, Ortiz JB, Kemmou S, Lifshitz J, Conrad CD \& Thomas TC. Early and persistent dendritic hypertrophy in the basolateral amygdala following experimental diffuse traumatic brain injury. Journal of Neurotrauma 2016 [in press]. (doi:10.1089/ neu.2015.4339)

72 van den Pol AN \& Gallyas F. Trauma-induced Golgi-like staining of neurons: a new approach to neuronal organization and response to injury. Journal of Comparative Neurology 1990296 654-673. (doi:10.1002/cne.902960410)

73 van den Pol AN. The magnocellular and parvocellular paraventricular nucleus of rat: intrinsic organization. Journal of Comparative Neurology 1982206 317-345. (doi:10.1002/cne.902060402)

74 Barry J. [Classification by the Golgi technique of several categories of neurons in the mouse paraventricular nucleus]. Comptes Rendus des Séances et Mémoires de la Société de Biologie et des ses Filiales 1975169 978-980.

75 Franzoni MF, Viglietti-Panzica C, Ramieri G \& Panzica GC. A Golgi study on the neuronal morphology in the hypothalamus of the Japanese quail (Coturnix coturnix japonica). I. Tuberal and mammillary regions. Cell and Tissue Research 1984236 357-364. (doi:10.1007/BF00214239)

Received in final form 16 May 2016

Accepted 17 June 2016

Accepted Preprint published online 17 June 2016 http://www.endocrineconnections.org DOI: 10.1530/EC-16-0031 (c) 2016 The authors Published by Bioscientifica Ltd.

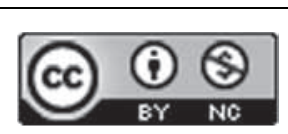

This work is licensed under a Creative Commons Attribution-NonCommercial 4.0 International License. 